\title{
Morphological Variation of Vegetative Phase among Ma Wee (Oryza sativa L.) Accessions during Non-Inductive Growing Season for Flowering
}

\author{
W.H.D.U Pushpakumari ${ }^{1}$, S. Geekiyanage ${ }^{2 *}$ \\ ${ }^{1}$ Faculty of Graduate Studies, University of Ruhuna, Sri Lanka \\ ${ }^{2}$ Department of Agricultural Biology, University of Ruhuna, Sri Lanka \\ *sudarshanee@Agbio.Ruh.Ac.Lk
}

\begin{abstract}
Ma wee (Oryza sativa L.) had been a popular traditional rice in the past due to desirable agronomic traits for adverse environments. Ma wee accessions (Accns) are known to be photoperiod sensitive for flowering initiation which flower during Maha (short day) season. Understanding morphological variation of Ma wee is useful in future analysis for genetic diversity. Thirty nine Accns of Ma wee varieties used for the present study with the objective of determining variation of morphological responses when Accns remained in vegetative phase under non-inductive photoperiod for flower initiation at 210 days after sowing. Thirty nine Accns from plant genetic resources center, Sri Lanka were grown in Kamburupitiya with four replicates in a completely randomised design at the end of Maha season in 2013/2014.
\end{abstract}

Quantitative characters of plant height $(\mathrm{PH})$, total tiller number (TTN), total leaf number (TLN), culm number (CN) and ligules length (LL) and qualitative characters of ligule color (LC), ligule shape (LS), internode color (IC), culm strength (CS) were recorded. None of the 39 Accns flowered by 210 days and PH, TTN, TLN, CN, and LL varied from $87.5 \pm 1.61 \mathrm{~cm}$, $2 \pm 0.37,8 \pm 0.84,2 \pm 0.43,1.8 \pm 0.46 \mathrm{~cm}$ to $199 \pm 1.36 \mathrm{~cm}, 22 \pm 2.09,136 \pm 2.20,24 \pm 2.03$ and $4.18 \pm 0.25 \mathrm{~cm}$ respectively. The lowest PH, TTN, TLN, and CN were recorded from Accn 6253. Except for Accn 6253, rests 38 Accns were over $1 \mathrm{~m}$ in PH.

Qualitative characters varied among clusters without any uniformity for a given cluster. CS varied among Accns from weak, intermediate to strong. LC and IC varied from green to white and green to yellow respectively. Acute to acuminate and two cleft LS were also observed. Principal Component Analysis followed by Cluster Analysis was performed using data from quantitative characters. First two of PCs explained over $80 \%$ of total morphological variations. The first PC represented the PH, TTN, TLN and CN whereas PC2 represented the LL. Eight clusters were obtained at rescaled distance five in the dendogram. Variation among Accns within clusters for qualitative characters may be an indication of genetic diversity.

Keywords: Ma wee, Morphological variation, Vegetative phase 